\title{
La planification sectorielle L'Opération sciences fondamentales*
}

\author{
SERGE LAPOINTE**
}

\section{RESUME}

L'auteur commence par décrire brièvement, en la situant dans son contexte, cette opération de planification qui portait sur un secteur de l'enseignement supérieur, celui des sciences fondamentales lequel comprenait comme sous-secteurs: la biochimie, la chimie, les mathématiques, la physique, les sciences biologiques et les sciences de la terre. Il prend le recul nécessaire pour dégager ce qui parait être en ce moment certains points forts et certains points faibles de l'entreprise de planification qu'est l'Opération sciences fondamentales. Enfin, il esquisse certaines interrogations ou certaines attentes qu'on peut nourrir à l'égard de la planification sectorielle de l'enseignement supérieur.

\section{ABSTRACT \\ Sectoral planning in the basic sciences}

The author begins by describing, from his own experience, the planning process for one area of higher education - the basic sciences, in which are included: biochemistry, chemistry, mathematics, physics, biological science, and earth science. He takes a perspective from which the current strengths and weaknesses of the planning process for the basic sciences may be seen. Lastly, he outlines particular questions and expectations that should be considered in regard to sectoral planning in higher education.

\section{Description de l'O.S.F.}

L'Opération sciences fondamentales, qu'on désigne souvent par le sigle O.S.F. a été lancée en juin 1973 après quelques mois de préparation générale et s'est terminée au début de cette année après une durée de deux ans et demi. Elle faisait suite à deux opérations de

\footnotetext{
*Communication présentée à la conference annuelle de la Société canadienne pour l'etude de l'enseignement supérieur, Québec, juin, 1976.

**Professeur titulaire, Université de Montréal (actuellement detaché à l'Université du Québec à Montréal).
} 
planification sectorielle analogues, l'Opération sciences appliquées ou O.S.A., entreprise au début de 1971 et qui se terminait lors du lancement de l'O.S.F. à l'été 1973, ainsi que l'Opération sciences de la santé ou O.S.S. lancée en juin 1972 et qui se termine présentement. L'Opération sciences appliquées a porté sur les divers génies, l'agriculture, la foresterie, la géodésie, la géologie et l'informatique. L'Opération sciences de la santé porte sur la médecine, la médecine dentaire et les nombreuses spécialités qui touchent à la santé. Ces trois opérations de planification ont été mises en marche par le ministère de l'Education, en collaboration avec le ministère des Affaires sociales dans le cas de l'O.S.S. Ce n'est certes pas mon intention de comparer ces trois études pour tenter d'en dégager les similitudes et les différences. Qu'il me suffise de dire que ces trois opérations ont été conçues à la même époque et qu'étant les premières études du genre au Québec, elles ont été élaborées suivant le même schéma général, la première l'O.S.A. exerçant son influence sur la deuxième I'O.S.S., et les deux premières servant de guide et d'inspiration à la troisième qui nous intéresse ici l'O.S.F. L'analogie, d'ailleurs, entre les activités scientifiques qu'on retrouve dans le champ de chacune de ces opérations de planification sectorielle ne pouvait que favoriser leur similitude. Toutefois l'objet propre de chaque opération ainsi que les préoccupations spécifiques des personnes qui y ont participé, ont conféré à chaque opération son identité particulière. C'est celle-là que je me propose de vous décrire maintenant en ce qui concerne l'O.S.F.

Globalement, le but de l'Opération sciences fondamentales était de prendre la mesure du secteur des sciences fondamentales et de proposer les objectifs et les moyens les plus aptes à favoriser le meilleur développement de ce secteur. Ce but s'impose par son évidence même. Il faut toutefois, pour pouvoir s'en approcher, fournir des réponses satisfaisantes à un certain nombre de questions préalables. La première tâche à accomplir est évidemment celle de définir et de circonscrire le secteur, épistémologiquement, empiriquement, administrativement. Il faut ensuite déterminer les données qui sont pertinentes pour l'inventaire: programmes, ressources, clientèles, débouchés. On ne peut éviter de se poser la question du rôle des sciences fondamentales dans la société. Enfin, dans notre société, compte tenu de ce rôle, il faut connaître le niveau de développement que l'on veut atteindre dans le secteur des sciences fondamentales. Nous reviendrons sur la plupart de ces points plus loin, lorsque nous tenterons d'apprécier les bonnes et les mauvaises fortunes de l'O.S.F. qui avait pour but, dans ce contexte, de proposer un plan quinquennal de développement pour la période 1976-1981 et un premier aperçu des tendances pour la période 1981-1986, afin de rationaliser et de coordonner les efforts des milieux universitaires.

En guise de commentaire sur ce qui précède, permettez-moi de citer un passage du premier document publié par l'O.S.F. et intitulé "But, Organisation, Programmation, Cheminement".

Il faut admettre sans ambages que les buts qui viennent d'être énoncés sont ambitieux et ne pourront sans doute etre atteints que d'une façon imparfaite. Par ailleurs, les moyens mis en oeuvre pour atteindre ces buts peuvent être considérés comme étant en eux-mêmes des buts secondaires de l'Opération sciences fondamentales. En effet, les divers inventaires sur les programmes de formation et de recherche, sur les débouchés et sur la clientèle étudiante seront un guide précieux pour tous les intéressés dans le secteur des sciences fondamentales. De plus, par une participation à l'élaboration d'un plan d'ensemble, l'Opération espère amener les professeurs et les adminis- 
trateurs du secteur à penser davantage en termes de priorités et de besoins à l'échelle du Québec, à formuler des objectifs communs de développement et à envisager des mécanismes de collaboration interinstitutionnelle afin que soit mieux orienté le dynamisme des individus et des institutions.

Pour terminer cette description sommaire de l'Opération sciences fondamentales, je décrirai maintenant brièvement le cadre dans lequel elle s'est déroulée. La première étape a consisté en la préparation par un Comité provisoire du ministère de l'Education d'un document de travail décrivant le but, l'organisation, la programmation et le cheminement de l'Opération. Ce document transmis pour étude à la direction de chaque université fut discuté à quelques reprises dans le cadre de la Conférence des recteurs et principaux des universités du Québec, par un groupe composé des vice-recteurs à la planification ou leur équivalent. Le document amendé à la suite de ces échanges, prévoyait la création de deux organismes: le Comité directeur de l'OSF et le Comité des sciences fondamentales. Le Comité directeur, nommé par le ministère de l'Education, comptait six membres dont quatre professeurs choisis dans autant d'universités et de disciplines que possible, un membre du personnel du Conseil des universités et, enfin, un membre de la Direction générale de l'enseignement supérieur du ministère de l'Education, ces deux derniers devant assurer la liaison avec les organismes dont ils étaient issus.

Par ailleurs les membres du Comité des sciences fondamentales ont été nommés par le Conseil des universités et devaient représenter autant que possible un large éventail de la communauté scientifique et des milieux intéressés par les sciences fondamentales. Ce comité, dans le cadre de l'Opération était consultatif: il avait pour rôle de commenter les documents qui lui étaient soumis par le Comité directeur portant sur les princiaples phases de l'Opération et, en particulier, les projets de rapport.

De son côté, le Comité directeur avait la responsabilité de la programmation des travaux, du choix des responsables de dossiers, de la coordination des études et, d'une façon générale, de la bonne marche de l'Opération. L'orientation des travaux d'analyse était du ressort du Comité directeur qui était également responsable de l'approbation des rapports et des recommandations, et de leur transmission aux autorités compétentes.

Par l'intermédiaire du Comité des sciences fondamentales du Conseil des universités, composé en bonne partie d'universitaires, le Comité directeur de L'O.S.F. a été en mesure de demeurer en contact avec certains courants de pensées du milieu universitaire concerné, tout au long de ses travaux. La participation recherchée des universités à l'élaboration d'un plan d'ensemble pour le secteur a pris également plusieurs autres formes.

Le champ de l'Opération sciences fondamentales a été divisé en sept sous-secteurs: biochimie, chimie, mathématiques, physique, sciences biologiques, sciences de la terre et un dernier sous-secteur qu'on a désigné sous le nom de domaines connexes et qui regroupait cinq domaines où les sciences fondamentales jouissent d'une bonne visibilité, l'environnement, la documentation scientifique, la traduction scientifique, le journalisme scientifique et la sociopolitique des sciences. Pour chacun des six premiers sous-secteurs et pour chacun des cinq domaines connexes, le Comité directeur a désigné un responsable, choisi pour sa compétence et dont le rôle était de monter un dossier contenant toutes les données pertinentes et présentant au Comité directeur un éclairage venant de l'intérieur sur les problèmes propres au sous-secteur ou au domaine en question. Dans tous les cas, sauf deux, journalisme et documentation scientifiques, les responsables étaient des professeurs d'uni- 
versité. De plus, le Comité directeur a convoqué à tous les trois mois environ des réunions de consultation regroupant les doyens de sciences et les doyens d'études avancées de chaque université pour recueillir les commentaires de ces derniers sur les principales phases des dossiers de sous-secteur, problématique, analyse, prospective et besoins, de même que sur un avant-projet de rapport.

Le Comité directeur a également commandité à divers membres de la communauté universitaire sept études particulières qui lui paraissaient importantes comme base du travail de planification en sciences fondamentales. Vers le milieu de ses travaux, en mai 1974, le Comité directeur organisait dans le cadre du congrès annuel de l'ACFAS un colloque de prospective auquel étaient conviés tous les membres de la communauté universitaire. Enfin après avoir distribué son projet de rapport dans les universités en septembre 1975, ces dernières ont été invitées à présenter un mémoire au Comité directeur et à le commenter lors d'audiences individuelles tenues à leur intention en novembre 1975.

\section{Points forts et points faibles de l'O.S.F.}

Les diverses activités qui viennent d'être énumérées brièvement ont suscité, à l'intérieur même des universités d'importants travaux de compilation, d'analyse et de réflexion reliés à la planification du secteur des sciences fondamentales. Cette participation du milieu universitaire à l'élaboration d'un plan d'ensemble, en somme le processus lui-même de l'Opération sciences fondamentales est peut-être la contribution positive la plus importante de celle-ci. ll s'agissait d'une démarche nouvelle à laquelle presque tous ont dû s'initier, y compris dans une large mesure les membres du Comité directeur eux-mêmes. S'il ne restait que cela dans les esprits de tous les intéressés, un souci de planification, une méthodologie qui ne peut aller qu'en s'améliorant, ce serait déjà beaucoup. On pourrait peut-être parler ici d'une planification préventive, par opposition à une planification curative, laquelle serait faite au fur et à mesure par les initiateurs mêmes des activités.

La deuxième contribution utile de l'O.S.F. serait d'avoir obtenu ou rendu disponibles un certain nombre de données. On songe bien sûr d'abord à un inventaire des programmes de formation aux premier, deuxième et troisième cycles, au relevé des clientèles étudiantes, à la présentation de l'évolution dans le temps du nombre de diplômés, à la description des effectifs d'enseignement et de recherche ainsi qu'à l'énumération des activités et des subventions de recherche. Ces renseignements étaient parfois connus à des degrés divers, mais ils ont pu, à cette occasion, être clarifiés, complétés et portés à la connaissance de tous. De plus, les données rassemblées ont pu être comparées dans certains cas à celles du reste du Canada et des Etats-Unis. Enfin, toujours au plan de l'information, le Comité directeur de l'O.S.F. a confié à des groupes de recherche trois grandes enquêtes: l'une portait sur l'attitude des étudiants au secondaire et au collégial à l'égard de la science et de la technologie, l'autre, menée auprès d'un groupe de diplômés, portait sur l'adéquation entre la formation reçue et les fonctions de travail, enfin une dernière, lancée auprès des quelques 19,000 diplômés en sciences fondamentales des universités québécoises, résidant au Québec, au Canada et ailleurs dans le monde, portait sur leur profil d'études et de carrière jusqu'à ce jour. Les résultats de ces trois études, tant qualitatifs que quantitatifs, n'existaient pas auparavant et sont sûrement de nature à éclairer ceux qui s'intéressent au secteur des sciences fondamentales. La dernière en particulier apporte les premiers éléments de réponse à la 
question autrefois assez embarrassante de savoir ce que font les diplômés en sciences fondamentales.

La troisième contribution de l'O.S.F. aura été de commencer à définir une problématique à partir des divers éléments recueillis. Toute démarche de planification suppose la reconnaissance de certaines valeurs, la détermination d'objectifs, l'élaboration et le choix de divers scénarios pour atteindre ces derniers compte tenu de la situation présente et de l'extrapolation dans le temps de ses tendances dynamiques. L'Opération sciences fondamentales est partie du postulat que l'existence d'un secteur des sciences fondamentales au Québec constituait une valeur acceptée de tous, comme dans toute société occidentale contemporaine. L'étape suivante supposait qu'on puisse déterminer, en termes qualitatifs et quantitatifs, les objectifs à atteindre dans le secteur des sciences fondamentales. Or il est bien évident que le niveau de développement qu'on souhaite donner à ce secteur est fonction du rôle que celui-ci est appelé à jouer dans l'ensemble de la société. Ce rôle est en partie connu: formation de personnel scientifique, d'enseignants. Cependant, cette affirmation est trompeuse. Combien veut-on de scientifiques, d'enseignants? Quelle est l'influence des sciences fondamentales dans la société et à quel niveau, compte tenu des objectifs que celle-ci veut poursuivre, faut-il fixer leur développement?

Cette question étant appelée à rester sans réponse pour l'instant, l'Opération sciences fondamentales a préféré tenter de définir les objectifs à poursuivre à partir de la notion de besoins de la société. En réalité, il s'agit là plus d'une option qui fut prise que d'une solution apportée au problème mentionné. Cette option consiste à reconnaitre l'intégration du secteur des sciences fondamentales dans l'ensemble du corps social. Bien sûr il importe de distinguer entre besoins exprimés par la société, et besoins inexprimés, entre besoins reconnus et besoins ignorés et enfin entre besoins définis et besoin d'une présence des sciences fondamentales dans l'ensemble des activités de la société. Cette optique ne résout pas pour autant la question du niveau souhaitable de développement. Dans ce but l'Opération sciences fondamentales, comme d'autres, a eu recours au critère de comparaison avec le reste du Canada et les Etat-Unis. Il est bien évident toutefois que la comparaison avec d'autres pays ne peut être qu'un indicateur de développement et ne peut à elle seule servir de norme. Ainsi, par exemple, on peut toujours se demander s'il faut plus ou moins de chimistes, de physiciens au Québec qu'ailleurs et pourquoi.

En fait, on touche là une des faiblesses de la planification sectorielle. Pour atteindre son but, celle-ci doit s'appuyer sur des éléments essentiels qui sont à l'extérieur de son propre champ. Ainsi, la logique voudrait que l'on possède une politique globale de développement scientifique avant de procéder à la planification du secteur des sciences fondamentales, ou une politique globale de l'enseignement supérieur avant de procéder à la planification d'un secteur de l'université, à défaut de quoi la démarche de planification ne peut en être qu'une de rationalisation des activités, et encore. A la défense de la planification sectorielle on pourrait dire toutefois que dans bien des cas attendre la formulation d'une politique globale pourrait retarder le développement rationnel du secteur en question. On peut même s'interroger sur la possibilité de réussir à formuler une politique globale de développement scientifique, par exemple, sans avoir au préalable procédé à l'inventaire et à l'analyse des secteurs scientifiques, et adopté des solutions pour le développement rationnel de chacun d'eux fondées sur leur dynamisme propre et leur logique interne.

Sans pouvoir déterminer de façon définitive le niveau de développement du secteur, 
l'O.S.F. a quand même pu privilégier l'atteinte de certains objectifs qualitatifs. Ainsi, tout en reconnaissant le rôle pour ainsi dire intrinsèque des sciences fondamentales qui est d'améliorer notre connaissance du monde, l'O.S.F. comme beaucoup d'autres a voulu insister sur l'intégration des sciences fondamentales et des scientifiques dans la société. A la suite d'autres auteurs, l'O.S.F. a qualifié le premier niveau d'activités, d'épistémologique et le second, d'opératoire. Certains ont voulu voir dans le niveau opératoire tel que conçu par l'O.S.F. une confusion entre les sciences fondamentales et les sciences appliquées. Il est à craindre que le débat là-dessus dure encore quelque temps, mais ce n'est pas l'endroit ni mon propos de l'aborder ici. Qu'il suffise de dire que le but visé en mettant l'insistance sur le niveau opératoire était de souligner l'importance croissante du rôle que jouent ou pourraient jouer les sciences fondamentales dans la société et aussi la nécessité pour beaucoup d'étudiants, compte tenu du nombre élevé de diplômés, et de leur profil de carrière probable, d'apprendre, en plus d'acquérir des connaissances de base, à transférer ces dernières dans des situations concrètes. Cette préoccupation se retrouve d'ailleurs dans le soin que l'O.S.F. a pris pour mettre en relief, à titre exemplaire, quelques domaines connexes aux sciences fondamentales.

La troisième contribution de l'O.S.F. que je viens de mentionner, soit l'élaboration d'une problématique et le choix de certaines options pourrait être qualifiée par certains de contribution mixte, c'est-à-dire à la fois bonne et mauvaise selon qu'on partage ou non les options prises. Toutefois, quelle que soit la valeur qu'on attribue à cette problématique, il est difficile de nier qu'elle vaut mieux qu'une absence de problématique en ce sens non pas qu'elle remplit un vide tant bien que mal, mais parce qu'elle donne prise à la réflexion et peut ainsi engendrer une meilleure compréhension de la situation.

Quant à l'objectif qui était de proposer un plan quinquennal de développement pour la période 1976-81 et un premier aperçu des tendances pour la période 1981-86, il faut bien admettre que c'est assez modestement qu'on s'en est rapproché. L'O.S.F. a recommandé que les universités réorientent progressivement tous leurs programmes dans le sens indiqué d'ici cinq ans et fassent rapport au fur et à mesure au Comité des programmes du Conseil des universités et de la Direction générale de l'enseignement supérieur; que toutes les mesures soient prises pour que le perfectionnement des maîtres soit terminé au plus tard dans dix ans. Après avoir insisté sur le principe de la complémentarité entre les universités, tant au niveau de l'enseignement que de la recherche, l'O.S.F. a recommandé qu'une opération soit entreprise et terminée au cours du premier plan quinquennal pour identifier et inventorier les divers champs de spécialisation en recherche en vue d'en arriver à une répartition plus rationnelle non seulement des activités actuelles mais surtout des nouvelles activités à développer. Plusieurs autres recommandations concrètes ont été faites qu'il serait déplacé d'énumérer ici. Mais on ne saurait parler d'un plan détaillé et rigoureusement articulé. Il est sans doute souhaitable qu'il en soit ainsi lorsqu'il s'agit de planification sectorielle de l'enseignement supérieur.

Le processus de planification théoriquement est un processus continu: les objectifs, une fois qu'ils sont atteints, deviennent la situation actuelle et il faut songer à fixer de nouveaux objectifs et à élaborer de nouveaux scénarios pour les atteindre. L'enseignement supérieur n'échappe pas à cette logique encore moins peut-être, du moins serait-on porté à le penser de nos jours, que d'autres secteurs d'activité qui semblent davantage suivre un cheminement dicté par la nécessité et caractérisé par l'inévitable. 
Pour illustrer le besoin qu'on ressent de planifier l'université, permettez-moi de terminer sur une note d'humour en citant un passage de La Cerisaie de Tchekov.

Le premier personnage dit:

Excusez-moi, mon cher, mais vous ne comprenez rien. S'il existe quelque chose d'intéressant, et même de remarquable dans toute la province, c'est bien notre cerisaie.

Le second personnage répond:

Ce jardin n'a de remarquable que son étendue. Il ne donne de cerises que tous les dix ans, et même alors, on ne sait qu'en faire, personne ne veut les acheter. 